females with an average age of 9 years old. The rural environment was found in 21 patients having close contact with dogs.

Clinically, 12 patients presented respiratory symptoms varying from simple dyspnoea and cough to hemoptysis and infectious signs. 8 patients reported only chest pain with no other signs. The diagnosis was accidentally in 3 cases. The pulmonary localization was found in 20 cases associated to hepatic hydatidosis in 8 cases. 2 patients had cerebral localization. The kidney and the pericardium were affected in 1 case each. The average size of the cysts was $7 \mathrm{~cm}$. Hydatic serology was positive in $73 \%$.

Computed tomography with contrast agent injection was performed in all the patients and allowed the diagnosis showing a simple cystic formation in 20 patients and multilocular cyst in 3 patients. It allowed also to look for second localization.

Treatment was based on the surgery in 95\% of cases with a medical treatment based on Albendazol in $78 \%$ for a period between 3 months and 2 years. A reoccurrence was noted in 1 case.

\section{P622 STAPHYLOCOCCIC NECROTIZING PNEUMONIA IN A TWO MONTHS INFANT}

Manel Charfi*, Amel Ben Hamad, Rim Abdelkrim, Chiraz Regaieg, Amira Bouraoui, Ridha Regaieg, Nedia Hmida, Afef Ben Thabet, abdellatif Gargouri. Department of Neonatology, Hedi Chaker University Hospital, Sfax, Tunisia

\subsection{6/archdischild-2019-epa.953}

Introduction Acute necrotizing pneumonia associated to Panton-Valentine Leukodine (PVL) producing staphylococcus aureus is a recent clinical entity identified by Gilet and all in 2002. It's a life threatening condition that can lead to rapid death despite a rapid management. It's rarely described on young infants less than one year old.

Methods We report the case of a two months old infant who was admitted in our unit for acute necrotizing pneumonia associated to PVL producing and Methicillin resistant Staphylococcus aureus.

Results A two months old boy with no past medical history was admitted in our unit for acute fever. His mother was nurse in our unit and his father was a nurse in the intensive care unit. Physical exam on admission showed high fever with tachycardia and acrocyanosis. Blood count was normal. The $\mathrm{C}$ reactive protein level was of $13 \mathrm{mg} / \mathrm{l}$. Chest radiography showed an infiltrate of the right lobe. Empiric antibiotic therapy with cefotaxim and vancomycin was started. Six hours after admission the infant developed cyanosis an acute dyspnea. Control chest radiography showed a huge pneumothorax. Mechanic ventilation was then started and a chest tube was set up. Drainage of the pleural cavity brought a purulent fluid. Bacteriological examination of the pleural fluid revealed a methicillin-resistant and a PVL producing Staphylococcus aureus. The same germ was found in the nasopharyngeal swab of the two parents as well as the infant claiming then an intrafamilial transmission. Clindamycin was added to the initial antibiotic therapy. Follow up chest radiography showed pneumatocele. The evolution was favorable. Chest tube was removed after 5 days. The infant was extubated a day after and he was dischaged after 4 weeks of triple antibioticc therapy. He is now 9 months old. His physical exam is normal as well as his chest radiography.

Conclusion The diagnosis of staphylococcic necrotizing pneumonia must be kept in mind every time one of these following signs is associated: viral infection preceding the onset, hemoptisis, rapid worsening, multilocular infiltrates, pneumatocele, pleural effusion or leucopenia. Moreover an association of anti-staphylococcal and antitoxin antibiotic must be started even before bacteriological confirmation.

\section{P623 THE CAUSE OF FEVER THAT SHOULD BE KEPT IN MIND IN FAMILY MEDICINE IN SETTLEMENTS WHERE LIVESTOCK FARMING IS WIDESPREAD: BRUCELLOSIS}

Çiğdem El*, Mehmet Emin Çelikkaya. Mustafa Kemal University, Hatay, Turkey

\subsection{6/archdischild-2019-epa.954}

Aim Brucellosis, which is primarily disease of animals, is also seen in humans in settlements where animal husbandry is common. Differential diagnosis is very important by reason of complaints and clinical findings of brucellosis are not specific. Brucellosis series in childhood are not reported commonly in the literature. The aim of this study is to retrospectively assesment the data of our brucellossis cases in childhood.

Material and method The data of the patients who were treated with the diagnosis of brucellosis between October2016 and October-2018 at Mustafa Kemal University,Department of Pediatrics, were retrospectively reviewed.The medical data that risk factors of brucella transmission (uncooked meat, milk and milk products consumption), demographic data, seasonal distribution, complaints of patients, time to diagnosis from onset of complaints, clinical and laboratory findings, family history were obtained.

Results The mean age of the patients in our study was 7.4 years (3-15). The mean time from the onset of symptoms to the diagnosis was 6 weeks (4-16). 78.87\% (n: 56) of the patients were acute and $21.12 \% \quad(\mathrm{n}: 15)$ of subacute brucellosis.

Clinical findings were 91.54\% (n:65), fever\% 87.32 (n:62), arthritis/arthralgia $\quad 90.14 \% \quad(\mathrm{n}: 64), \quad$ hepatomegaly $\quad 19.71 \%$ (n:14), splenomegaly $9.85 \%$ (n:7), petechia-purpura was $2.8 \%$ $(\mathrm{n}: 2)$, scrotal swelling and redness $1.4 \%(\mathrm{n}: 1)$.

During the first evaluation of the patients, the Wright agglutination test $85.91 \%(\mathrm{n}: 61)$ found to be positive. During initial assessment for wright agglutination test was negative in 10 patients despite who supported the diagnosis of brucellosis with complaints, family history, brucella transmission risk factors and clinical findings. However when repeated wright agglutination test on these patients after one week the results were positive. Brucellosis family history was found in $52.11 \%$ (n:37) of the patients.

Discussion and conclussion There is difficulty in the diagnosis of brucellosis infections which is still a serious public health problem because of the lack of diagnostic clinical and laboratory findings. Although with early diagnosis and response to treatment of the brucellosis are very well, unfortunately late diagnosis and inadequate treatment may be causes mortality and morbidity with serious complications. Brucellosis, which an endemic disease common in our country should be kept in 
mind in the diagnosis of children patients who had complaints that fever, weakness, weight loss and especially joint pain, where pediatric clinics.

\section{P624 HOSPITAL-ACQUIRED NEONATAL MENINGITIS: EXPERIENCE OF THE NEONATOLOGY DEPARTEMET OF SFAX (TUNISIA)}

${ }^{1}$ Amel Ben Hamed, ${ }^{1}$ Adel Babay, ${ }^{1}$ Manel Charfi, ${ }^{2}$ Nour Houda Ben Ayed, ${ }^{1}$ Chiraz Regaieg*, ${ }^{1}$ Amira Bouraoui, ${ }^{1}$ Nedia Hmida, ${ }^{2}$ Adnene Hamami, ${ }^{1}$ Afef Ben Thabet, ${ }^{1}$ Abdellatif Gargouri. ${ }^{1}$ Department of Neonatology, Hedi Chaker Hospital, Sfax, Tunisia; ${ }^{2}$ Microbiology Laboratory, Habib Bourguiba Hospital, Sfax, Tunisia

10.1136/archdischild-2019-epa.955

Background The past decade brought with it both highly sophisticated neonatal intensive care with improved perinatal mortality rates and increased risk for nosocomial septicemia and meningitis among survivors. Although most of these infections were caused by multiple antibiotic-resistant bacteria. The absence of specific clinical signs makes diagnosis of meningitis more difficult.

Methods We enrolled neonates who were admitted to neonatal unit at at Hedi Chaker hospital, Sfax from 1 January 2007 to 31 December 2016 and had suspected nosocomial infection with abnormal cerebrospinal fluid examination.

Results Five cases were reviewed, Among these 4 were girls (80\%), All neonates were preterm between 27 and 34 weeks of pregnancy, with Low birth weight in 2 cases and very Low birth weight in 3 cases, the average age of diagnosis was 14.2 days after hospitalization, $4(80 \%)$ of them had concomitant bacteremia, suspected bacterial infection was confirmed in 4 cases $(80 \%)$, by positive results on blood culture in 4 cases but only one cerebrospinal fluid (CSF) culture was positive, Klebsiella pneumoniae was the leading pathogen. The case mortality rate (CMR) was 0.8 . Four patients died and one case was cured but he developed a sequellae of psychomotoric retardation.

Conclusion Preterm neonates have a high risk of developing nosocomial infections and especially Nosocomial Meningitis wich is related to a high case mortality rate, Definitive diagnosis is made by cerebrospinal fluid examination (CSF) via lumbar puncture (LP), which should be performed in any neonate suspected of having sepsis or meningitis. Future efforts should be directed toward better definition of bacterial virulence, host susceptibility and preventive measures.

\section{P625 IMPACT OF ANTIBACTERIAL DRUGS' OVERUSE IN DURATION OF HOSPITALIZATION AMONG PATIENTS WITH ACUTE DIARRHEAL DISEASES IN 'NORK' ICH, ARMENIA, 2018}

\footnotetext{
1,2,3 Erik Grigoryan, ${ }^{4}$ Mark Grigoryan, 4,5Vigen Asoyan, 4,5,6 Hripsime Apresyan*. ${ }^{1}$ Armenian State University of Economics, Yerevan, Armenia; ${ }^{2}$ Swiss UMEF University, Yerevan, Armenia; ${ }^{3}$ Amberd Research Center, Yerevan, Armenia; ${ }^{4}$ Yerevan State Medical University after Mkhitar Heratsi, Yerevan, Armenia; ${ }^{5}$ Muratsan University Hospital Complex, Yerevan, Armenia; ${ }^{6}$ Wigmore Clinic, Yerevan, Armenia
}

\subsection{6/archdischild-2019-epa.956}

Background Antibiotics were announced as life-savers when they became widely available in the middle of 20th century. Nowadays, antimicrobials are fast becoming killers themselves. The more any given antibiotic is used, the greater the chances that bacteria will develop antimicrobial resistance (AMR) that renders the drug ineffective.

Although we don't have exact number of deaths from antibiotic-resistant infections in Armenia, we give importance to its prediction and prevention of developing multidrug resistant strains of bacteria. Our goal is to describe the low cost-effectiveness of treatment in accordance with national and international guidelines to hospitalized patients with acute diarrheal diseases. It is an option why not to use antibiotics inappropriate.

Objective The purpose of this study was to evaluate the costeffectiveness of treatment with national guidelines and so to create a premise for prevention of inappropriate antibiotic use.

Methods A retrospective economic analysis was performed using 45-day (01.04.-15.05.2018) data from "Nork" ICH. The analysis was conducted from a third-party payer's perspective.

During the above mentioned period 156 patients were admitted from which $125(80.1 \%)$ had watery and $31(19.9 \%)$ bloody diarrhea. Antibacterial treatment was given to 80 patients, but only in $23(28.75 \%)$ cases it was indicated. Widely used antibacterials were nifuroxazide $55.6 \%$, azithromycin 4.4\%, amoxicillin 1.1\%, metronidazole 3.3\%, ciprofloxacin 13.3\%, TMP-SMX $6.7 \%$, cefotaxime $1.1 \%$, ceftriaxone $14.5 \%$.

Patients with appropriate antibacterial treatment were hospitalized 6.8 days on average. The patients without any antibacterial treatment and patients with inappropriate antibacterial treatment were hospitalized 4.7 and 6.3 days on average respectively.

Average Total Cost of treatment was calculated as follows:

$\mathrm{a}-$ average duration of hospitalization $b^{*}$ - average cost of treatment per patient per day

* - it is fixed cost - 36.7EUR

Eventually, average costs of treatments have been determined.

Results Since average cost of treatment is known- 36.7EUR per patient per day, totally 172.49EUR per patient, it can be compared with inappropriate treatment cost-totally 231.21EUR per patient. The difference in the costs of two treatment options is 58.72 EUR per patient. Multiplying by the number (57) of patients managed with inappropriate treatment we will have total difference 3347EUR during 45 days.

Conclusions Treatment in accordance with national guidelines is cheaper in comparison with inappropriate antibacterial treatment as the last requires longer duration of hospitalization.

The implementation of guidelines is important from both clinical and financial points of view.

\section{P626 INFLAMMATORY MARKERS OF ANTIPSYCHOTIC WEIGHT GAIN AND CARDIOMETABOLIC DYSFUNCTION IN YOUTH MENTAL HEALTH DISORDERS}

\footnotetext{
1,2Karen Conlan, ${ }^{3,4}$ Eadaoin Hayes*, 'Louise Gallagher, ${ }^{4}$ Nicole Wood, ${ }^{5}$ Andrew Hogan, ${ }^{3}$ Donal O'Shea, 'Jane McGrath. 'Trinity College, Dublin, Ireland; ${ }^{2}$ National Children's Research Centre, Dublin; ${ }^{3}$ University College Dublin, Dublin, Ireland; ${ }^{4}$ National Children's Research Centre, Dublin, Ireland; ${ }^{5}$ NUI Maynooth, Kildare, Ireland
}

\subsection{6/archdischild-2019-epa.957}

Introduction Second generation antipsychotics (SGAs) are prescribed to treat mental health disorders in children. However, there are concerns about these medications due to 